\title{
Los pacientes quieren acceder a sus historias clínicas electrónicas
}

\author{
Patients want to access their electronic medical records
}

Delbanco T y col. Annals of Internal Medicine 2012; 157:461-470

\section{Objetivos}

Evaluar el efecto que produce en médicos y pacientes el hecho de permitirle a estos últimos leer las evoluciones escritas por los médicos en sus historias clínicas.

\section{Diseño}

Estudio antes-después no controlado.

\section{Lugar y Pacientes}

Tres centros de atención primaria en Estados Unidos. Se invito a participar a todos los médicos de atención primaria afiliados a los centros participantes.

\section{Intervención}

Se invitó a los médicos participantes a ofrecer acceso a las historias clínicas electrónicas de todos sus pacientes.Se realizaron encuestas electrónicas a médicos y pacientes antes y después de plantear la posibilidad de acceso a sus registros.

\section{Medición de Resultados Principales}

Los resultados principales se obtuvieron de las encuestas previamente detalladas.

\section{Resultados Principales}

De los 113 médicos que participaron, 105 (93\%) completaron e estudio y 99 respondieron la encuesta pre y post intervención. Experiencia de los pacientes: Del total de pacientes invitados, solo 5.391(41\%) abrieron al menos una vez una evolución medica y completaron la encuesta post-intervención. De estos entre el $77 \%$ y el $87 \%$ sintieron que estaban mas cuidados en relación a su salud; $60 \%$ al $78 \%$ reportaron un aumento en la adherencia a su medicación crónica; $26 \%$ al $36 \%$ comentaron algún problema de confidencialidad; y 1 al $8 \%$ reportaron confusión, preocupación o se sintieron ofendidos por algún registro medico.
Entre el 59 y $62 \%$ de los pacientes comento la necesidad de agregar comentarios a las evoluciones de los médicos. Uno de cada tres pacientes creía que ellos deberían aprobar el contenido de las evoluciones.

Al final del estudio el $99 \%$ de los pacientes quiso continuar con la intervención.

Experiencia de los médicos: Luego de la intervención pocos médicos reportaron consultas mas extensas (0 a $5 \%$ ) 0 tomarse mas tiempo para responder preguntas por fuera de la consulta (0 a $8 \%$ ).

Del 3 al $36 \%$ de los médicos reportaron cambios en su forma de documentar las evoluciones y entre el 0 y el $21 \%$ afirmaron tomarse más tiempo para registrar.

85 a $96 \%$ de los médicos acordaron con la opinión de los pacientes en relación a la necesidad de aprobación del contenido de las evoluciones.

Ningún médico eligió suspender la intervención.

El volumen de mensajes entre médicos y pacientes no se modifico.

\section{Conclusiones}

Los pacientes accedieron a sus evoluciones frecuentemente, la mayoría reporto beneficios clínicos relevantes y muy pocas preocupaciones y el $99 \%$ de los pacientes quisieron continuar con el estudio. Los médicos reportaron un efecto bajo sobre su flujo habitual de trabajo lo que indicaría que valdría la pena continuar con el acceso de los pacientes a sus historias clínicas.

Fuentes de financiamiento: Robert Wood Johnson Foundation, Drane Family Fund, Richard and Florence Koplow Charitable Foundation y National Cancer Institute.

Conflicto de interés de los autores: no reportado

\section{Comentario}

El presente estudio es parte de una serie de estudios promovidos por el proyecto "OpenNotes", una iniciativa de Estados Unidos que invita a los pacientes a leer su historia clínica'. Los autores eligieron un diseño cuasi experimental (sin aleatorización de los participantes ni grupo control) lo cual podría afectar la validez del estudio². Además, optaron solo por conocer las percepciones de médicos y pacientes y no realizaron medición directa sobre resultados clínicos o de procesos, lo que hubiera aumentado la significancia del estudio.
Además, la invitación a participar a los pacientes, fue de forma voluntaria lo que podría haber aumentado la respuesta positiva por parte de los participantes. Finalmente, vale la pena destacar la baja tasa de respuesta de la encuesta por parte de los pacientes, hecho que podría interferir con la generalización de los resultados. Si bien ya se han publicado experiencias similares $^{3,4}$, este es el primer estudio que describe una experiencia en más de un centro de salud, donde se evaluó además la percepcion de médicos y pacientes en el mismo estudio.

Damián Borbolla [ Informática Medica. Hospital Italiano de Buenos Aires. damian.borbolla@ hospitalitaliano.org.ar ]

Borbolla D. Los pacientes quieren acceder a sus historias clínicas electrónicas. Evid Act Pract Ambul. Jul-Sep 2013;16(3):85. Comentado de: Delbanco T, Walker J, Bell SK, et al. Inviting patients to read their doctors' notes: a quasi-experimental study and a look ahead. Ann. Intern. Med. 2012 Oct 2;157(7):461-70. PMID: 23027317

\section{Referencias}

1. Delbanco T, Walker J, Darer JD, Elmore JG, Feldman HJ, Leveille SG, et al. Open notes: doctors and patients signing on. Ann. Intem. Med. 2010 Jul 20;153(2):121-5.

2. Harris AD, McGregor JC, Perencevich EN, Furuno JP, Zhu J, Peterson DE, et al. The use and interpretation of quasi-experimental studies in medical informatics. J Am Med Inform Assoc. 2006 Feb;13(1):16-23.

3. Woods SS, Schwartz E, Tuepker A, Press NA, Nazi KM, Turvey CL, et al. Patient Experiences With Full Electronic Access to Health Records and Clinical Notes Through the My HealtheVet Personal Health Record Pilot: Qualitative Study. J. Med. Intemet Res. 2013;15(3):e65.

4. Zulman DM, Nazi KM, Turvey CL, Wagner TH, Woods SS, An LC. Patient interest in sharing personal health record information: a web-based survey. Ann. Intern. Med. 2011 Dec 20;155(12):805-10. 